\title{
Solvability of Neumann boundary value problem for fractional $p$-Laplacian equation
}

\section{Bo Zhang*}

\section{"Correspondence:}

zhangbohuaibei@163.com

School of Mathematical Sciences,

Huaibei Normal University, Huaibei,

235000, P.R. China

\begin{abstract}
We consider the existence of solutions for a Neumann boundary value problem for the fractional $p$-Laplacian equation. Under certain nonlinear growth conditions of the nonlinearity, we obtain a new result on the existence of solutions by using the continuation theorem of coincidence degree theory.

MSC: 34A08; 34B15

Keywords: Neumann boundary value problem; fractional differential equation; p-Laplacian operator; continuation theorem
\end{abstract}

\section{Introduction}

The purpose of this paper is to establish the existence of solutions for the following Neumann boundary value problem (NBVP for short) for a fractional $p$-Laplacian equation:

$$
\left\{\begin{array}{l}
D_{0^{+}}^{\beta} \phi_{p}\left(D_{0^{+}}^{\alpha} x(t)\right)=g(t, x(t)), \quad t \in[0, T], \\
D_{0^{+}}^{\alpha} x(0)=D_{0^{+}}^{\alpha} x(T)=0,
\end{array}\right.
$$

where $0<\alpha, \beta \leq 1, D_{0^{+}}^{\alpha}$ is a Caputo fractional derivative, $\phi_{p}(s)=|s|^{p-2} s(p>1), T>0$ is a given constant and $g:[0, T] \times \mathbb{R} \rightarrow \mathbb{R}$ is continuous. Obviously, $\phi_{p}$ is invertible and its inverse operator is $\phi_{q}$, where $q>1$ is a constant such that $1 / p+1 / q=1$.

The fractional calculus is a generalization of the ordinary differentiation and integration on an arbitrary order that can be noninteger. Fractional differential equations appear in a number of fields such as physics, polymer rheology, regular variation in thermodynamics, biophysics, blood flow phenomena, aerodynamics, electro-dynamics of a complex medium, viscoelasticity, Bode analysis of feedback amplifiers, capacitor theory, electrical circuits, electro-analytical chemistry, biology, control theory, fitting of experimental data, etc. (see [1-4]). In recent years, because of the intensive development of the fractional calculus theory itself and its applications, fractional differential equations have been of great interest. For example, Agarwal et al. (see [5]) considered a two-point boundary value problem at nonresonance, and Bai (see [6]) considered a $m$-point boundary value problem at resonance. For more papers on fractional boundary value problems, see [7-15] and the references therein.

In [7], by using the coincidence degree theory for Fredholm operators, the authors studied the existence of solutions for the following NBVP:

$$
\left\{\begin{array}{l}
D_{0^{+}}^{\beta} \phi_{p}\left(D_{0^{+}}^{\alpha} x(t)\right)=f\left(t, x(t), D_{0^{+}}^{\alpha} x(t)\right), \quad t \in[0,1], \\
D_{0^{+}}^{\alpha} x(0)=D_{0^{+}}^{\alpha} x(1)=0 .
\end{array}\right.
$$

o 2015 Zhang; licensee Springer. This is an Open Access article distributed under the terms of the Creative Commons Attribution License (http://creativecommons.org/licenses/by/4.0), which permits unrestricted use, distribution, and reproduction in any medium, provided the original work is properly credited. 
Notice that $D_{0^{+}}^{\beta} \phi_{p}\left(D_{0^{+}}^{\alpha}\right)$ is a nonlinear operator, so it is not a Fredholm operator. Hence, there is a bug in the proof of the main result.

\section{Preliminaries}

In this section, for convenience of the reader, we will present here some necessary basic knowledge and definitions as regards the fractional calculus theory, which can be found, for instance, in $[2,4]$.

Definition 2.1 The Riemann-Liouville fractional integral operator of order $\alpha>0$ of a function $u:(0,+\infty) \rightarrow \mathbb{R}$ is given by

$$
I_{0^{+}}^{\alpha} u(t)=\frac{1}{\Gamma(\alpha)} \int_{0}^{t}(t-s)^{\alpha-1} u(s) d s
$$

provided that the right-side integral is pointwise defined on $(0,+\infty)$.

Definition 2.2 The Caputo fractional derivative of order $\alpha>0$ of a continuous function $u:(0,+\infty) \rightarrow \mathbb{R}$ is given by

$$
\begin{aligned}
D_{0^{+}}^{\alpha} u(t) & =I_{0^{+}}^{n-\alpha} \frac{d^{n} u(t)}{d t^{n}} \\
& =\frac{1}{\Gamma(n-\alpha)} \int_{0}^{t}(t-s)^{n-\alpha-1} u^{(n)}(s) d s,
\end{aligned}
$$

where $n$ is the smallest integer greater than or equal to $\alpha$, provided that the right-side integral is pointwise defined on $(0,+\infty)$.

Lemma 2.1 (see [1]) Let $\alpha>0$. Assume that $u, D_{0^{+}}^{\alpha} u \in L([0, T], \mathbb{R})$. Then the following equality holds:

$$
I_{0^{+}}^{\alpha} D_{0^{+}}^{\alpha} u(t)=u(t)+c_{0}+c_{1} t+\cdots+c_{n-1} t^{n-1},
$$

where $c_{i} \in \mathbb{R}, i=0,1, \ldots, n-1$, here $n$ is the smallest integer greater than or equal to $\alpha$.

Lemma 2.2 (see [16]) For any $u, v \geq 0$, then

$$
\begin{aligned}
& \phi_{p}(u+v) \leq \phi_{p}(u)+\phi_{p}(v), \quad \text { if } p<2 ; \\
& \phi_{p}(u+v) \leq 2^{p-2}\left(\phi_{p}(u)+\phi_{p}(v)\right), \quad \text { if } p \geq 2 .
\end{aligned}
$$

Now we briefly recall some notations and an abstract existence result, which can be found in [17].

Let $X, Y$ be real Banach spaces, $L: \operatorname{dom} L \subset X \rightarrow Y$ be a Fredholm operator with index zero, and $P: X \rightarrow X, Q: Y \rightarrow Y$ be projectors such that

$$
\begin{aligned}
& \operatorname{Im} P=\operatorname{Ker} L, \quad \operatorname{Ker} Q=\operatorname{Im} L, \\
& X=\operatorname{Ker} L \oplus \operatorname{Ker} P, \quad Y=\operatorname{Im} L \oplus \operatorname{Im} Q .
\end{aligned}
$$


It follows that

$$
\left.L\right|_{\operatorname{dom} L \cap \operatorname{Ker} P}: \operatorname{dom} L \cap \operatorname{Ker} P \rightarrow \operatorname{Im} L
$$

is invertible. We denote the inverse by $K_{P}$.

If $\Omega$ is an open bounded subset of $X$ such that $\operatorname{dom} L \cap \bar{\Omega} \neq \varnothing$, then the map $N: X \rightarrow Y$ will be called $L$-compact on $\bar{\Omega}$ if $Q N(\bar{\Omega})$ is bounded and $K_{P}(I-Q) N: \bar{\Omega} \rightarrow X$ is compact.

Lemma 2.3 (see [17]) Let $X$ and $Y$ be two Banach spaces, $L: \operatorname{dom} L \subset X \rightarrow Y$ be a Fredholm operator with index zero, $\Omega \subset X$ be an open bounded set, and $N: \bar{\Omega} \rightarrow Y$ be L-compact on $\bar{\Omega}$. Suppose that all of the following conditions hold:

(1) $L x \neq \lambda N x, \forall x \in \partial \Omega \cap \operatorname{dom} L, \lambda \in(0,1)$;

(2) $Q N x \neq 0, \forall x \in \partial \Omega \cap \operatorname{Ker} L$;

(3) $\operatorname{deg}(J Q N, \Omega \cap \operatorname{Ker} L, 0) \neq 0$, where $J: \operatorname{Im} Q \rightarrow \operatorname{Ker} L$ is an isomorphism map.

Then the equation $L x=N x$ has at least one solution on $\bar{\Omega} \cap \operatorname{dom} L$.

\section{Main result}

In this section, we will give the main result on the existence of solutions for NBVP (1.1).

Theorem 3.1 Let $g:[0, T] \times \mathbb{R} \rightarrow \mathbb{R}$ be continuous. Assume that

$\left(\mathrm{C}_{1}\right)$ there exists a constant $d>0$ such that

$$
(-1)^{i} u g(t, u)>0 \quad(i=1,2), \forall t \in[0, T],|u|>d
$$

$\left(\mathrm{C}_{2}\right)$ there exist nonnegative functions $a, b \in C[0, T]$ such that

$$
|g(t, u)| \leq a(t)|u|^{p-1}+b(t), \quad \forall t \in[0, T], u \in \mathbb{R} .
$$

Then NBVP (1.1) has at least one solution, provided that

$$
\begin{aligned}
& \gamma_{1}:=\frac{2^{p-1} T^{\beta+\alpha p-\alpha}\|a\|_{0}}{\Gamma(\beta+1)(\Gamma(\alpha+1))^{p-1}}<1, \quad \text { if } p<2 ; \\
& \gamma_{2}:=\frac{2^{2 p-3} T^{\beta+\alpha p-\alpha}\|a\|_{0}}{\Gamma(\beta+1)(\Gamma(\alpha+1))^{p-1}}<1, \quad \text { if } p \geq 2 .
\end{aligned}
$$

For making use of the continuation theorem to study the existence of solutions for NBVP (1.1), we consider the following system:

$$
\left\{\begin{array}{l}
D_{0^{+}}^{\alpha} x_{1}(t)=\phi_{q}\left(x_{2}(t)\right), \\
D_{0^{+}}^{\beta} x_{2}(t)=g\left(t, x_{1}(t)\right), \\
x_{2}(0)=x_{2}(T)=0 .
\end{array}\right.
$$

Clearly, if $x(\cdot)=\left(x_{1}(\cdot), x_{2}(\cdot)\right)^{\mathrm{T}}$ is a solution of NBVP $(3.2)$, then $x_{1}(\cdot)$ must be a solution of NBVP (1.1). Hence, to prove that NBVP (1.1) has solutions, it suffices to show that NBVP (3.2) has solutions.

In this paper, we take $X=\left\{x=\left(x_{1}, x_{2}\right)^{\mathrm{T}} \mid x_{1}, x_{2} \in C[0, T]\right\}$ with the norm $\|x\|=\max \left\{\left\|x_{1}\right\|_{0}\right.$, $\left.\left\|x_{2}\right\|_{0}\right\}$, where $\left\|x_{i}\right\|_{0}=\max _{t \in[0, T]}\left|x_{i}(t)\right|(i=1,2)$. By means of the linear functional analysis theory, we can prove $X$ is a Banach space. 
Define the operator $L: \operatorname{dom} L \subset X \rightarrow X$ by

$$
L x=\left(\begin{array}{c}
D_{0^{+}}^{\alpha} x_{1} \\
D_{0^{+}}^{\beta} x_{2}
\end{array}\right)
$$

where

$$
\operatorname{dom} L=\left\{x \in X \mid D_{0^{+}}^{\alpha} x_{1}, D_{0^{+}}^{\beta} x_{2} \in C[0, T], x_{2}(0)=x_{2}(T)=0\right\} .
$$

Let $N: X \rightarrow X$ be the Nemytskii operator

$$
N x(t)=\left(\begin{array}{c}
\phi_{q}\left(x_{2}(t)\right) \\
g\left(t, x_{1}(t)\right)
\end{array}\right), \quad \forall t \in[0, T] .
$$

Then NBVP (3.2) is equivalent to the operator equation as follows:

$$
L x=N x, \quad x \in \operatorname{dom} L
$$

Next we will give some lemmas which are useful in the proof of Theorem 3.1.

Lemma 3.1 Let L be defined by (3.3), then

$$
\begin{aligned}
& \operatorname{Ker} L=\left\{x \in X \mid x(t)=c \in \mathbb{R}^{2}, \forall t \in[0, T]\right\}, \\
& \operatorname{Im} L=\left\{y \in X \mid y_{1}(0)=0, \int_{0}^{T}(T-s)^{\beta-1} y_{2}(s) d s=0\right\} .
\end{aligned}
$$

Proof Obviously, from Lemma 2.1, (3.5) holds.

If $y \in \operatorname{Im} L$, then there exists $x \in \operatorname{dom} L$ such that $y=L x$. That is, $y_{1}(t)=D_{0^{+}}^{\alpha} x_{1}(t), y_{2}(t)=$ $D_{0^{+}}^{\beta} x_{2}(t)$. By Lemma 2.1, we have

$$
x_{2}(t)=c+\frac{1}{\Gamma(\beta)} \int_{0}^{t}(t-s)^{\beta-1} y_{2}(s) d s, \quad c \in \mathbb{R} .
$$

From the boundary value conditions $D_{0^{+}}^{\alpha} x_{1}(0)=x_{2}(0)=x_{2}(T)=0$, we obtain

$$
y_{1}(0)=0, \quad \int_{0}^{T}(T-s)^{\beta-1} y_{2}(s) d s=0 .
$$

So we get (3.6).

On the other hand, suppose $y \in X$ which satisfies (3.7). Let $x_{1}(t)=I_{0^{+}}^{\alpha} y_{1}(t), x_{2}(t)=$ $I_{0^{+}}^{\beta} y_{2}(t)$. Clearly $x_{2}(0)=x_{2}(T)=0$. Hence $x=\left(x_{1}, x_{2}\right)^{\mathrm{T}} \in \operatorname{dom} L$ and $L x=y$. Thus $y \in \operatorname{Im} L$. The proof is completed.

Lemma 3.2 Let $L$ be defined by (3.3), then $L$ is a Fredholm operator of index zero. The linear projectors $P: X \rightarrow X$ and $Q: X \rightarrow X$ can be defined as

$$
\begin{aligned}
& P x(t)=x(0), \quad \forall t \in[0, T] \\
& Q y(t)=\left(\begin{array}{c}
y_{1}(0) \\
\frac{\beta}{T^{\beta}} \int_{0}^{T}(T-s)^{\beta-1} y_{2}(s) d s
\end{array}\right):=\left(\begin{array}{c}
(Q y)_{1}(t) \\
(Q y)_{2}(t)
\end{array}\right), \quad \forall t \in[0, T] .
\end{aligned}
$$


Furthermore, the operator $K_{P}: \operatorname{Im} L \rightarrow \operatorname{dom} L \cap \operatorname{Ker} P$ can be written by

$$
K_{P} y=\left(\begin{array}{c}
I_{0^{+}}^{\alpha} y_{1} \\
I_{0^{+}}^{\beta} y_{2}
\end{array}\right) .
$$

Proof For any $y \in X$, we have

$$
\begin{aligned}
Q^{2} y(t) & =Q\left(\begin{array}{c}
y_{1}(0) \\
(Q y)_{2}(t)
\end{array}\right) \\
& =\left(\begin{array}{c}
y_{1}(0) \\
(Q y)_{2}(t) \cdot \frac{\beta}{T^{\beta}} \int_{0}^{T}(T-s)^{\beta-1} d s
\end{array}\right)=Q y(t) .
\end{aligned}
$$

Let $y^{*}=y-Q y$, then we get $y_{1}^{*}(0)=0$ and

$$
\begin{aligned}
& \int_{0}^{T}(T-s)^{\beta-1} y_{2}^{*}(s) d s \\
& \quad=\int_{0}^{T}(T-s)^{\beta-1} y_{2}(s) d s-\int_{0}^{T}(T-s)^{\beta-1}(Q y)_{2}(s) d s \\
& \quad=\frac{T^{\beta}}{\beta}\left((Q y)_{2}(t)-\left(Q^{2} y\right)_{2}(t)\right)=0 .
\end{aligned}
$$

So $y^{*} \in \operatorname{Im} L$. Thus $X=\operatorname{Im} L+\operatorname{Im} Q$. Since $\operatorname{Im} L \cap \operatorname{Im} Q=\{0\}$, we have $X=\operatorname{Im} L \oplus \operatorname{Im} Q$. Hence

$$
\operatorname{dim} \operatorname{Ker} L=\operatorname{dim} \operatorname{Im} Q=\operatorname{codim} \operatorname{Im} L=2 .
$$

This means that $L$ is a Fredholm operator of index zero.

For $y \in \operatorname{Im} L$, from the definition of $K_{P}$, we have

$$
L K_{P} y=\left(\begin{array}{c}
D_{0^{+}}^{\alpha} I_{0^{+}}^{\alpha} y_{1} \\
D_{0^{+}}^{\beta} I_{0^{+}}^{\beta} y_{2}
\end{array}\right)=y .
$$

On the other hand, for $x \in \operatorname{dom} L \cap \operatorname{Ker} P$, we get $x_{1}(0)=x_{2}(0)=0$. By Lemma 2.1, we obtain

$$
K_{P} L x=\left(\begin{array}{l}
x_{1}-x_{1}(0) \\
x_{2}-x_{2}(0)
\end{array}\right)=x
$$

So we know that $K_{P}=\left(L_{\operatorname{dom} L \cap \operatorname{Ker} P}\right)^{-1}$. The proof is completed.

Lemma 3.3 Let $N$ be defined by (3.4). Assume $\Omega \subset X$ is an open bounded subset such that $\operatorname{dom} L \cap \bar{\Omega} \neq \varnothing$, then $N$ is L-compact on $\bar{\Omega}$.

Proof By the continuity of $\phi_{q}$ and $g$, we find that $Q N(\bar{\Omega})$ and $K_{P}(I-Q) N(\bar{\Omega})$ are bounded. Moreover, there exists a constant $A>0$ such that $\|(I-Q) N x\| \leq A, \forall x \in \bar{\Omega}, t \in[0, T]$. Hence, in view of the Arzelà-Ascoli theorem, we need only to prove that $K_{P}(I-Q) N(\bar{\Omega}) \subset$ $X$ is equicontinuous. 
For $0 \leq t_{1}<t_{2} \leq T, x \in \bar{\Omega}$, we have

$$
\begin{aligned}
& K_{P}(I-Q) N x\left(t_{2}\right)-K_{P}(I-Q) N x\left(t_{1}\right) \\
& \quad=\left(\begin{array}{c}
I_{0^{+}}^{\alpha}((I-Q) N x)_{1}\left(t_{2}\right)-I_{0^{+}}^{\alpha}((I-Q) N x)_{1}\left(t_{1}\right) \\
I_{0^{+}}^{\beta}((I-Q) N x)_{2}\left(t_{2}\right)-I_{0^{+}}^{\beta}((I-Q) N x)_{2}\left(t_{1}\right)
\end{array}\right) .
\end{aligned}
$$

From $\|(I-Q) N x\| \leq A, \forall x \in \bar{\Omega}, t \in[0, T]$, we can see that

$$
\begin{aligned}
\mid I_{0^{+}}^{\alpha}( & (I-Q) N x)_{1}\left(t_{2}\right)-I_{0^{+}}^{\alpha}((I-Q) N x)_{1}\left(t_{1}\right) \mid \\
= & \frac{1}{\Gamma(\alpha)} \mid \int_{0}^{t_{2}}\left(t_{2}-s\right)^{\alpha-1}((I-Q) N x)_{1}(s) d s \\
& \quad-\int_{0}^{t_{1}}\left(t_{1}-s\right)^{\alpha-1}((I-Q) N x)_{1}(s) d s \mid \\
\leq & \frac{A}{\Gamma(\alpha)}\left\{\int_{0}^{t_{1}}\left[\left(t_{1}-s\right)^{\alpha-1}-\left(t_{2}-s\right)^{\alpha-1}\right] d s+\int_{t_{1}}^{t_{2}}\left(t_{2}-s\right)^{\alpha-1} d s\right\} \\
= & \frac{A}{\Gamma(\alpha+1)}\left[t_{1}^{\alpha}-t_{2}^{\alpha}+2\left(t_{2}-t_{1}\right)^{\alpha}\right] .
\end{aligned}
$$

Since $t^{\alpha}$ is uniformly continuous on $[0, T]$, we can obtain that $\left(K_{P}(I-Q) N(\bar{\Omega})\right)_{1} \subset C[0, T]$ is equicontinuous. A similar proof can show that $\left(K_{P}(I-Q) N(\bar{\Omega})\right)_{2} \subset C[0, T]$ is also equicontinuous. Hence, we find that $K_{P}(I-Q) N: \bar{\Omega} \rightarrow X$ is compact. The proof is completed.

Lemma 3.4 Suppose $\left(\mathrm{C}_{1}\right),\left(\mathrm{C}_{2}\right)$ hold, then the set

$$
\Omega_{1}=\{x \in \operatorname{dom} L \mid L x=\lambda N x, \lambda \in(0,1)\}
$$

is bounded.

Proof For $x \in \Omega_{1}$, we have $N x \in \operatorname{Im} L$. Thus, from (3.6), we obtain

$$
\int_{0}^{T}(T-s)^{\beta-1} g\left(s, x_{1}(s)\right) d s=0 .
$$

Then, by the integral mean value theorem, there exists a constant $\xi \in(0, T)$ such that $g\left(\xi, x_{1}(\xi)\right)=0$. So, from $\left(C_{1}\right)$, we get $\left|x_{1}(\xi)\right| \leq d$. By Lemma 2.1 , we have

$$
x_{1}(t)=x_{1}(\xi)-I_{0^{+}}^{\alpha} D_{0^{+}}^{\alpha} x_{1}(\xi)+I_{0^{+}}^{\alpha} D_{0^{+}}^{\alpha} x_{1}(t)
$$

which together with

$$
\begin{aligned}
\left|I_{0^{+}}^{\alpha} D_{0^{+}}^{\alpha} x_{1}(t)\right| & =\frac{1}{\Gamma(\alpha)}\left|\int_{0}^{t}(t-s)^{\alpha-1} D_{0^{+}}^{\alpha} x_{1}(s) d s\right| \\
& \leq \frac{1}{\Gamma(\alpha)}\left\|D_{0^{+}}^{\alpha} x_{1}\right\|_{0} \cdot \frac{1}{\alpha} t^{\alpha} \\
& \leq \frac{T^{\alpha}}{\Gamma(\alpha+1)}\left\|D_{0^{+}}^{\alpha} x_{1}\right\|_{0^{\prime}}, \quad \forall t \in[0, T]
\end{aligned}
$$


and $\left|x_{1}(\xi)\right| \leq d$ yields

$$
\left\|x_{1}\right\|_{0} \leq d+\frac{2 T^{\alpha}}{\Gamma(\alpha+1)}\left\|D_{0^{+}}^{\alpha} x_{1}\right\|_{0} .
$$

By $L x=\lambda N x$, we have

$$
\left\{\begin{array}{l}
D_{0^{+}}^{\alpha} x_{1}(t)=\lambda \phi_{q}\left(x_{2}(t)\right) \\
D_{0^{+}}^{\beta} x_{2}(t)=\lambda g\left(t, x_{1}(t)\right)
\end{array}\right.
$$

From the first equation of (3.9), we get $x_{2}(t)=\lambda^{1-p} \phi_{p}\left(D_{0^{+}}^{\alpha} x_{1}(t)\right)$. Then, by substituting it to the second equation of (3.9), we get

$$
D_{0^{+}}^{\beta} \phi_{p}\left(D_{0^{+}}^{\alpha} x_{1}(t)\right)=\lambda^{p} g\left(t, x_{1}\right):=\lambda^{p} N_{g} x_{1}(t) .
$$

Thus, from Lemma 2.1 and the boundary value condition $x_{2}(0)=0$, we obtain

$$
\phi_{p}\left(D_{0^{+}}^{\alpha} x_{1}(t)\right)=\lambda^{p} I_{0^{+}}^{\beta} N_{g} x_{1}(t) .
$$

So, from $\left(\mathrm{C}_{2}\right)$, we have

$$
\begin{aligned}
\left|\phi_{p}\left(D_{0^{+}}^{\alpha} x_{1}(t)\right)\right| & \leq \frac{1}{\Gamma(\beta)} \int_{0}^{t}(t-s)^{\beta-1}\left|g\left(s, x_{1}(s)\right)\right| d s \\
& \leq \frac{1}{\Gamma(\beta)} \int_{0}^{t}(t-s)^{\beta-1}\left(a(s)\left|x_{1}(s)\right|^{p-1}+b(s)\right) d s \\
& \leq \frac{T^{\beta}}{\Gamma(\beta+1)}\left(\|a\|_{0}\left\|x_{1}\right\|_{0}^{p-1}+\|b\|_{0}\right), \quad \forall t \in[0, T],
\end{aligned}
$$

which together with $\left|\phi_{p}\left(D_{0^{+}}^{\alpha} x_{1}(t)\right)\right|=\left|D_{0^{+}}^{\alpha} x_{1}(t)\right|^{p-1}$ and (3.8) yields

$$
\left\|D_{0^{+}}^{\alpha} x_{1}\right\|_{0}^{p-1} \leq \frac{T^{\beta}}{\Gamma(\beta+1)}\left[\|b\|_{0}+\|a\|_{0}\left(d+\frac{2 T^{\alpha}}{\Gamma(\alpha+1)}\left\|D_{0^{+}}^{\alpha} x_{1}\right\|_{0}\right)^{p-1}\right] .
$$

If $p<2$, by Lemma 2.2, we get

$$
\begin{aligned}
\left\|D_{0^{+}}^{\alpha} x_{1}\right\|_{0}^{p-1} & \leq \frac{T^{\beta}}{\Gamma(\beta+1)}\left[\|b\|_{0}+\|a\|_{0}\left(d^{p-1}+\frac{\left(2 T^{\alpha}\right)^{p-1}}{(\Gamma(\alpha+1))^{p-1}}\left\|D_{0^{+}}^{\alpha} x_{1}\right\|_{0}^{p-1}\right)\right] \\
& =A_{1}+\frac{2^{p-1} T^{\beta+\alpha p-\alpha}\|a\|_{0}}{\Gamma(\beta+1)(\Gamma(\alpha+1))^{p-1}}\left\|D_{0^{+}}^{\alpha} x_{1}\right\|_{0}^{p-1}
\end{aligned}
$$

where $A_{1}=\frac{T^{\beta}}{\Gamma(\beta+1)}\left(\|b\|_{0}+d^{p-1}\|a\|_{0}\right)$. Then, from (3.1), we have

$$
\left\|D_{0^{+}}^{\alpha} x_{1}\right\|_{0} \leq\left(\frac{A_{1}}{1-\gamma_{1}}\right)^{q-1}:=B_{1} .
$$

Thus, from (3.8), we get

$$
\left\|x_{1}\right\|_{0} \leq d+\frac{2 T^{\alpha}}{\Gamma(\alpha+1)} B_{1} .
$$


If $p \geq 2$, similar to the above argument, we let $A_{2}=\frac{T^{\beta}}{\Gamma(\beta+1)}\left(\|b\|_{0}+2^{p-2} d^{p-1}\|a\|_{0}\right)$, we obtain

$$
\left\|x_{1}\right\|_{0} \leq d+\frac{2 T^{\alpha}}{\Gamma(\alpha+1)} B_{2}
$$

where $B_{2}=\left(\frac{A_{2}}{1-\gamma_{2}}\right)^{q-1}$. Hence, combining (3.10) with (3.11), we have

$$
\left\|x_{1}\right\|_{0} \leq \max \left\{d+\frac{2 T^{\alpha}}{\Gamma(\alpha+1)} B_{1}, d+\frac{2 T^{\alpha}}{\Gamma(\alpha+1)} B_{2}\right\}:=B
$$

From the second equation of (3.9), Lemma 2.1, and $x_{2}(0)=0$, we have

$$
x_{2}(t)=\lambda I_{0^{+}}^{\beta} N_{g} x_{1}(t)
$$

So we have

$$
\left\|x_{2}\right\|_{0} \leq \frac{T^{\beta}}{\Gamma(\beta+1)} G_{B}
$$

where $G_{B}=\max \{|g(t, x)||t \in[0, T]| x \mid, \leq B\}$. Thus, from (3.12), we obtain

$$
\|x\|=\max \left\{\left\|x_{1}\right\|_{0},\left\|x_{2}\right\|_{0}\right\} \leq \max \left\{B, \frac{T^{\beta}}{\Gamma(\beta+1)} G_{B}\right\}:=M .
$$

Hence, $\Omega_{1}$ is bounded. The proof is completed.

Lemma 3.5 Suppose $\left(\mathrm{C}_{1}\right)$ holds, then the set

$$
\Omega_{2}=\{x \in \operatorname{Ker} L \mid Q N x=0\}
$$

is bounded.

Proof For $x \in \Omega_{2}$, we have $x_{1}(t)=c_{1}, x_{2}(t)=c_{2}, \forall t \in[0, T], c_{1}, c_{2} \in \mathbb{R}$, and

$$
\begin{aligned}
& \phi_{q}\left(c_{2}\right)=0, \\
& \int_{0}^{T}(T-s)^{\beta-1} g\left(s, c_{1}\right) d s=0 .
\end{aligned}
$$

From (3.13), we get $c_{2}=0$. From (3.14) and $\left(\mathrm{C}_{1}\right)$, we get $\left|c_{1}\right| \leq d$. Thus, we have

$$
\|x\|=\max \left\{\left|c_{1}\right|,\left|c_{2}\right|\right\} \leq d
$$

Hence, $\Omega_{2}$ is bounded. The proof is completed.

Lemma 3.6 Suppose $\left(\mathrm{C}_{1}\right)$ holds, then the set

$$
\Omega_{3}=\{x \in \operatorname{Ker} L \mid \mu x+(1-\mu) J Q N x=0, \mu \in[0,1]\}
$$


is bounded, where $J: \operatorname{Im} Q \rightarrow \operatorname{Ker} L$ defined by

$$
J\left(x_{1}, x_{2}\right)^{\mathrm{T}}=\left((-1)^{i} x_{2}, x_{1}\right)^{\mathrm{T}}
$$

is an isomorphism map.

Proof For $x \in \Omega_{3}$, we have $x_{1}(t)=c_{1}, x_{2}(t)=c_{2}, \forall t \in[0, T], c_{1}, c_{2} \in \mathbb{R}$, and

$$
\begin{aligned}
& \mu c_{1}+(-1)^{i}(1-\mu) \frac{\beta}{T^{\beta}} \int_{0}^{T}(T-s)^{\beta-1} g\left(s, c_{1}\right) d s=0, \\
& \mu c_{2}+(1-\mu) \phi_{q}\left(c_{2}\right)=0 .
\end{aligned}
$$

From (3.16), we get $c_{2}=0$ because $c_{2}$ and $\phi_{q}\left(c_{2}\right)$ have the same sign. From (3.15), if $\mu=0$, we get $\left|c_{1}\right| \leq d$ because of $\left(C_{1}\right)$. If $\mu \in(0,1]$, we can also get $\left|c_{1}\right| \leq d$. In fact, if $\left|c_{1}\right|>d$, in view of $\left(C_{1}\right)$, one has

$$
\mu c_{1}^{2}+(1-\mu) \frac{\beta}{T^{\beta}} \int_{0}^{T}(T-s)^{\beta-1}(-1)^{i} c_{1} g\left(s, c_{1}\right) d s>0,
$$

which contradicts to (3.15). So $\|x\| \leq d$. Hence, $\Omega_{3}$ is bounded. The proof is completed.

\section{Proof of Theorem 3.1 Set}

$$
\Omega=\{x \in X \mid\|x\|<\max \{M, d\}+1\} .
$$

Obviously $\left(\Omega_{1} \cup \Omega_{2} \cup \Omega_{3}\right) \subset \Omega$. It follows from Lemmas 3.2 and 3.3 that $L$ (defined by (3.3)) is a Fredholm operator of index zero and $N$ (defined by (3.4)) is $L$-compact on $\bar{\Omega}$. Moreover, by Lemmas 3.4 and 3.5, the conditions (1) and (2) of Lemma 2.3 are satisfied. Hence, it remains to verify the condition (3) of Lemma 2.3. Define the operator $H: \bar{\Omega} \times$ $[0,1] \rightarrow X$ by

$$
H(x, \mu)=\mu x+(1-\mu) J Q N x .
$$

Then, from Lemma 3.6, we have

$$
H(x, \mu) \neq 0, \quad \forall(x, \mu) \in(\partial \Omega \cap \operatorname{Ker} L) \times[0,1] .
$$

Thus, by the homotopy property of the degree, we have

$$
\begin{aligned}
\operatorname{deg}(J Q N, \Omega \cap \operatorname{Ker} L, \theta) & =\operatorname{deg}(H(\cdot, 0), \Omega \cap \operatorname{Ker} L, \theta) \\
& =\operatorname{deg}(H(\cdot, 1), \Omega \cap \operatorname{Ker} L, \theta) \\
& =\operatorname{deg}(I, \Omega \cap \operatorname{Ker} L, \theta) \neq 0,
\end{aligned}
$$

where $\theta$ is the zero element of $X$. So the condition (3) of Lemma 2.3 is satisfied.

Consequently, by Lemma 2.3, the operator equation $L x=N x$ has at least one solution $x(\cdot)=\left(x_{1}(\cdot), x_{2}(\cdot)\right)^{\mathrm{T}}$ on $\bar{\Omega} \cap \operatorname{dom} L$. Namely, NBVP (1.1) has at least one solution $x_{1}(\cdot)$. The proof is completed. 


\section{Competing interests}

The author declares that he has no competing interests.

\section{Acknowledgements}

The author is grateful for the valuable comments and suggestions of the referees.

Received: 20 October 2014 Accepted: 19 December 2014 Published online: 05 March 2015

\section{References}

1. Kilbas, AA, Srivastava, HM, Trujillo, JJ: Theory and Applications of Fractional Differential Equations. Elsevier, Amsterdam (2006)

2. Podlubny, I: Fractional Differential Equations. Academic Press, San Diego (1999)

3. Sabatier, J, Agrawal, OP, Machado, JAT (eds.): Advances in Fractional Calculus: Theoretical Developments and Applications in Physics and Engineering. Springer, Dordrecht (2007)

4. Samko, SG, Kilbas, AA, Marichev, Ol: Fractional Integrals and Derivatives: Theory and Applications. Gordon \& Breach, Yverdon (1993)

5. Agarwal, RP, O'Regan, D, Stanek, S: Positive solutions for Dirichlet problems of singular nonlinear fractional differential equations. J. Math. Anal. Appl. 371, 57-68 (2010)

6. Bai, Z: On solutions of some fractional $m$-point boundary value problems at resonance. Electron. J. Qual. Theory Differ. Equ. 2010, 37 (2010)

7. Chen, T, Liu, W, Hu, Z: A boundary value problem for fractional differential equation with $p$-Laplacian operator at resonance. Nonlinear Anal. TMA 75, 3210-3217 (2012)

8. Chen, T, Liu, W: An anti-periodic boundary value problem for the fractional differential equation with a $p$-Laplacian operator. Appl. Math. Lett. 25, 1671-1675 (2012)

9. Benchohra, M, Hamani, S, Ntouyas, SK: Boundary value problems for differential equations with fractional order and nonlocal conditions. Nonlinear Anal. TMA 71, 2391-2396 (2009)

10. Bai, Z, Lü, H: Positive solutions for boundary value problem of nonlinear fractional differential equation. J. Math. Anal. Appl. 311, 495-505 (2005)

11. Darwish, MA, Ntouyas, SK: On initial and boundary value problems for fractional order mixed type functional differential inclusions. Comput. Math. Appl. 59, 1253-1265 (2010)

12. El-Shahed, M, Nieto, JJ: Nontrivial solutions for a nonlinear multi-point boundary value problem of fractional order. Comput. Math. Appl. 59, 3438-3443 (2010)

13. Jiang, W: The existence of solutions to boundary value problems of fractional differential equations at resonance. Nonlinear Anal. TMA 74, 1987-1994 (2011)

14. Kosmatov, N: A boundary value problem of fractional order at resonance. Electron. J. Differ. Equ. 2010, 135 (2010)

15. Su, X: Boundary value problem for a coupled system of nonlinear fractional differential equations. Appl. Math. Lett. $22,64-69(2009)$

16. Lian, H: Boundary Value Problems for Nonlinear Ordinary Differential Equations on Infinite Intervals. Doctoral thesis (2007)

17. Gaines, R, Mawhin, J: Coincidence Degree and Nonlinear Differential Equations. Springer, Berlin (1977)

\section{Submit your manuscript to a SpringerOpen ${ }^{\circ}$ journal and benefit from:}

- Convenient online submission

- Rigorous peer review

Immediate publication on acceptance

- Open access: articles freely available online

- High visibility within the field

- Retaining the copyright to your article 